\title{
Application of Improved SOM Neural Network in Manufacturing Process Quality Control
}

\author{
Yibing Li \\ School of Mechanic and Electronic Engineering \\ Wuhan University of Technology \\ Wuhan, China, 430070 \\ ahlyb@163.com
}

\author{
Fei Pan \\ School of Mechanic and Electronic Engineering \\ Wuhan University of Technology \\ Wuhan, China, 430070 \\ pfwhlgdx@163.com
}

\begin{abstract}
The use of neural networks in quality control has been a popular research topic over the last decade. An adaptive self-organizing mapping (SOM) neural network algorithm is proposed to overcome the shortages of traditional neural networks in this paper. In order to improve the classification effectiveness of SOM neural network, this paper designs an improved SOM neural network, which improved the algorithm formula based on input vector, the number setting of competitive layer neurons and the initializing weight vector. And the method is used to classify the product of cement slide shoe bearing in manufacturing process quality control, and experiment results show that the algorithm adapts well the unsupervised learning problems.
\end{abstract}

Keywords-Self-organizing Mapping (SOM), artificial neural network, manufacturing process quality control

\section{INTRODUCTION}

Quality control of manufacturing process is the essential parts for ensuring product high quality. Under advanced manufacturing paradigm, it is the first task and precondition of quality management and control to monitor process status effectively, which can detect timely and eliminate the affection of the abnormal manufacturing process, and enables the manufacturing process operating status asymptotically stable and satisfies the given process performance index.

There are a number of quality characteristics that need to be monitored and controlled simultaneously in manufacturing process. The methods of statistical process control (SPC) are recognized as one of the most important tools for monitoring all these characteristics simultaneously [1]. However, the effectiveness of SPC is strictly dependent on statistical assumptions and unable to quantify and visualized the process status data. For considering the question of noise tolerance, some research focus on the combination between SPC and artificial neural networks (ANNs).

The other broad type of ANNs are those with non supervised training, such as the well-known Self-Organizing Map, SOM which provides a topology-preserving mapping from a high-dimensional input space onto a two-dimensional map space. So, the methods of statistical learning (SL) can be used in the process control to avoid these shortages. An adaptive self-organizing mapping (SOM) neural network algorithm is proposed to overcome the shortages of traditional neural networks, and enhance the monitoring of manufacturing process in this paper. Finally, the performances of SOM neural network is further evaluated in the cement slide shoe bearing manufacturing process. The experimental results indicate that the algorithm adapts well the unsupervised learning problems.

\section{IMPROVED SOM NeURAL NETWORK MODEL}

\section{A. Model of SOM}

The most distinguishing feature of self-organizing mapping (SOM) is the fact that it's an un-supervised clustering method, and the network topology shown in figure1. The model includes the input layer and the competition layer, and every neurons of input layer can be combined with competition layer by weightings. The neurons number is determined by the components number of input vectors, the neurons of output layer was arranged in a plane according to a certain way, and the most common is square and hexagons. When the input signals are received by input layer, the output layer will be inspired and excited to determine the category of input info.

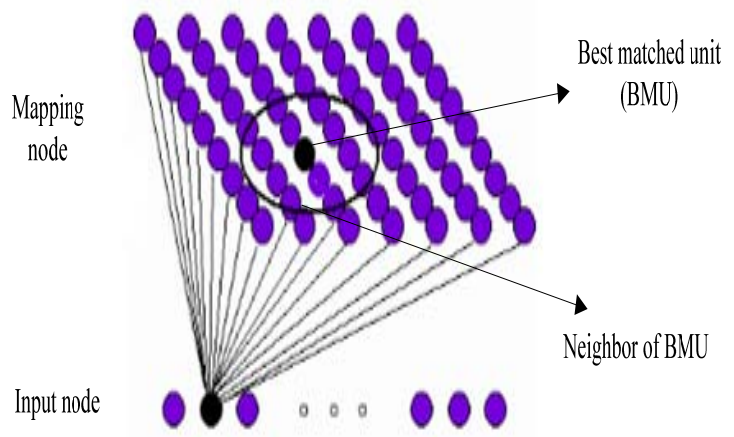

Figure 1. Model of SOM

\section{B. Training algorithm of SOM}

Online clustering is carried out in the input pattern, and any expectation output cannot be given in the learning process of SOM. When the input vector is received by SOM, the neurons of competition layer obtain the responses from input pattern by competition, and find the best matched unit (BUM) which is the smallest distance with input samples. And the connect weight can be mapped the input vector by 
adjusting the winner neurons and adjacent connect weight. Finally every neuron is divided into distinct areas that has different response characteristic to the same input vector, it realizes the clustering.

The training algorithm steps of SOM are as follows:

Step1: Initializing the weight vector. And every weight of output layer is assigned and normalized by random number, the weight vector describes as $W_{j}, \mathrm{j}=1,2, \ldots$

Setp2: Receiving the input vector from training sets, and describing as $X_{i}=\left\{x_{1}, x_{2}, \ldots, x_{n}\right\}$.

Sep3: Finding out the winner neuron of competition layer. The neuron is defined the winner neurons which has the smallest distances between $X_{i}$ and $W_{j}$.

Step4: Adjusting the weight by formula (1).

$W_{i j}=W_{i j}+\eta\left(X_{i}-W_{i j}\right)$

There, $W_{i j}$ means the weight between input layer node $\mathrm{i}$ and output layer node $\mathrm{j}$, and the learning rate is signed as $\eta$ which is decreased as training numbers.

Step 5: Checking at the end of the iteration. The training of SOM can be finished by judging whether the learning rate is equal to zero or the given value, or the training time achieves the set value or not. Otherwise, return to step 2 and go on.

\section{Improved algorithm of SOM based on genetic algorithms}

SOM learning method is based on the self-learning method as the core of the "winner takes all" rules[2]. When the input vector is inputted into the SOM network, we can find the winning neuron by calculating weights, which also called the best matched unit (BMU). Then adjust the weights by the neighborhood contraction learning factor method, and make the connection weights to form a group that can be mapped input vector data. As can be seen from the works of the SOM neural network, the key factors which affect the SOM network training speed and accuracy not only have a close relationship with the SOM heuristic algorithm, input vector, competitive layer topology and initial weights of SOM networks.

Therefore, the improved training algorithm steps are as follows:

Step 1: initialization. Each neuron $\mathrm{j}$ of SOM is expressed by an $\mathrm{n}$ dimensional vector $w_{j}=\left[w_{j 1}, w_{j 2}, \ldots, w_{j n}\right]$, the nearest neighbor neurons represent the topology of neurons connected by some kind of relationship.

Step 2: In the training process, the input vector $x=\left[x_{1}, x_{2}, \ldots x_{n}\right]$ is input into the SOM network.

Step 3: Calculate the distance between $\mathrm{x}$ and each neuron of SOM network, which is by calculating the distance of the vector and the weight vector $w$, to find the winning neuron BMU. Define $w_{j^{*}}$ as winning neuron, $d_{j^{*}}$ is the Euclidean distance between the input vector and the winning neuron weight vector $w$, so

$$
d_{j^{*}}=\left\|x-w_{j^{*}}\right\|=\sqrt{\sum_{i=1}^{n}\left(x_{i}-w_{i j}\right)^{2}}
$$

Step 4: After find the winning neuron, update the weights of the winning neuron $w_{j^{*}}$ and its neighboring,

$$
w_{i j}(t+1)=w_{i j}(t)+\eta(t) \times h_{j, i}(x)(t) \times\left(x_{i}-w_{i j}(t)\right)
$$

where $\mathrm{t}$ is the number of times of learning, $\eta(t)$ is the learning rate function, $h_{j i}(x)(t)$ is field function.

$$
\begin{aligned}
& \eta(t)=\eta_{0} \exp \left\{-\frac{t}{\tau}\right\} \\
& h_{j i}(x)(t)=\exp \left\{-\frac{\left\|r_{j}-r_{i}\right\|_{2}}{2 \sigma^{2}(t)}\right\}
\end{aligned}
$$

where $\eta_{0}$ is initial value, $\tau$ is exponential decay time constant, $r_{i}$ is the discrete position of winning neural units $i$. $r_{i}$ is the position of excitatory neurons j. $\sigma(t)$ is width parameter, define $\sigma_{0}$ as initial value. Then,

$$
\sigma(t)=\sigma_{0} \times\left(1-\frac{t}{\tau}\right)
$$

Step 5: When $t$ is equal to the learning number $\mathrm{T}$, the learning process stops and complete the independent study, otherwise returns to step 2 .

\section{APPLICATION ANALYSIS IN THE CEMENT SLIDE SHOE BEARING MANUFACTURING PROCESS BASED ON SOM}

Products classification and diagnosis of defections can have intuitive understanding of the quality of products, facilitating the adjustment to improve the manufacturing process, and also improving the pass rate of products. In this paper, we take the cement slide shoe bearing for example.

About 30 sample data sets are selected from manufacturing process of the cement slide shoe bearing. Every sample contains 7 features according to Table 1 . Respectively indicated by P1-P7, specific samples are listed in Table 1.

Table 1 Sample Characteristics

\begin{tabular}{cccccccc}
\hline \multirow{2}{*}{$\begin{array}{c}\text { Sa- } \\
\text { mple }\end{array}$} & \multicolumn{7}{c}{ parameter } \\
\cline { 2 - 8 } X1 & 1449.7 & 850.22 & 460.153 & 130.02 & 412.94 & 0.53 & 35.5 \\
X2 & 1449.0 & 850.22 & 460.255 & 120.00 & 412.96 & 0.58 & 38.3 \\
X3 & 1449.3 & 850.30 & 460.141 & 130.02 & 413.00 & 0.74 & 37.5 \\
X4 & 1449.8 & 850.24 & 460.244 & 129.94 & 412.94 & 0.72 & 39.5 \\
X5 & 1449.3 & 850.30 & 460.188 & 130.04 & 412.94 & 0.68 & 36.7 \\
X6 & 1449.6 & 850.34 & 460.210 & 130.00 & 412.92 & 0.62 & 39.3 \\
X7 & 1449.2 & 850.38 & 460.202 & 130.02 & 413.04 & 0.74 & 35.1 \\
X8 & 1449.0 & 850.36 & 460.233 & 129.94 & 412.94 & 0.51 & 38.9 \\
X9 & 1449.1 & 850.38 & 460.238 & 130.04 & 413.08 & 0.78 & 38.6 \\
X10 & 1449.7 & 850.32 & 460.150 & 129.98 & 412.94 & 0.65 & 39.5 \\
X11 & 1450.0 & 850.20 & 460.171 & 130.06 & 412.94 & 0.57 & 38.5
\end{tabular}




\begin{tabular}{llllllll} 
X12 & 1449.4 & 850.24 & 460.192 & 130.08 & 412.96 & 0.79 & 35.2 \\
X13 & 1449.4 & 850.36 & 460.249 & 130.06 & 413.08 & 0.67 & 38.7 \\
X14 & 1449.8 & 850.26 & 460.162 & 130.00 & 412.98 & 0.66 & 37.5 \\
X15 & 1449.2 & 850.24 & 460.158 & 129.98 & 413.08 & 0.65 & 39.5 \\
X16 & 1449.5 & 850.32 & 460.156 & 129.96 & 413.10 & 0.69 & 38.0 \\
X17 & 1449.4 & 850.26 & 460.244 & 130.00 & 412.98 & 0.70 & 38.1 \\
X18 & 1449.8 & 850.32 & 460.172 & 129.98 & 412.94 & 0.57 & 37.4 \\
X19 & 1449.7 & 850.34 & 460.206 & 130.00 & 412.96 & 0.61 & 39.0 \\
X20 & 1449.8 & 850.36 & 460.157 & 130.06 & 412.98 & 0.80 & 37.9 \\
X21 & 1449.3 & 850.30 & 460.242 & 130.04 & 413.02 & 0.51 & 35.9 \\
X22 & 1449.7 & 850.22 & 460.215 & 129.98 & 412.96 & 0.77 & 36.2 \\
X23 & 1449.7 & 850.26 & 460.182 & 130.06 & 413.02 & 0.77 & 39.4 \\
X24 & 1449.2 & 850.38 & 460.202 & 130.02 & 413.04 & 0.74 & 35.1 \\
X25 & 1449.1 & 850.24 & 460.188 & 129.98 & 412.94 & 0.53 & 37.4 \\
X26 & 1449.5 & 850.38 & 460.149 & 130.10 & 412.92 & 0.58 & 35.8 \\
X27 & 1450.0 & 850.32 & 460.169 & 130.08 & 412.96 & 0.60 & 39.9 \\
X28 & 1449.3 & 850.40 & 460.155 & 130.00 & 412.96 & 0.70 & 38.6 \\
X29 & 1449.6 & 850.22 & 460.162 & 130.02 & 412.98 & 0.54 & 37.5 \\
X30 & 1449.2 & 850.24 & 460.169 & 130.02 & 413.00 & 0.72 & 37.4 \\
\hline
\end{tabular}

The first 20 sets of data are used to train the SOM network, the last 10 sets of data are used to test its. Considering the uniform of unit of data, normalization is performed firstly in this paper. In addition, the topology structure of SOM network is supposed to be a hexagonal structure. Competitive layer has $6 * 6=36$ neurons, and distance function, classification learning rate, neighborhood distance of tuning stage etc take the default value, training for 200 times. Top twenty sets of data for network learning, output clustering results through Matlab simulation as Table 2

Table 2 Classification result of network after training

\begin{tabular}{ccccccccccc}
\hline Columns & $\mathbf{1}$ & $\mathbf{2}$ & $\mathbf{3}$ & $\mathbf{4}$ & $\mathbf{5}$ & $\mathbf{6}$ & $\mathbf{7}$ & $\mathbf{8}$ & $\mathbf{9}$ & $\mathbf{1 0}$ \\
Clustering results & 31 & 7 & 9 & 1 & 6 & 24 & 34 & 20 & 25 & 2 \\
Columns & $\mathbf{1 1}$ & $\mathbf{1 2}$ & $\mathbf{1 3}$ & $\mathbf{1 4}$ & $\mathbf{1 5}$ & $\mathbf{1 6}$ & $\mathbf{1 7}$ & $\mathbf{1 8}$ & $\mathbf{1 9}$ & $\mathbf{2 0}$ \\
Clustering results & 15 & 12 & 27 & 38 & 4 & 4 & 13 & 36 & 35 & 22 \\
\hline Columns & represent & the & first & twenty & sets & of data
\end{tabular}

Columns represent the first twenty sets of data, data belongs. Blue neurons represents winning neuron in Fig.2, number indicate the number of winning neuron. Coordinate $(0,0)$ corresponding class 1 neuron, Coordinate $(1,0)$ corresponding class 2 neuron, and so on. In Fig. 3, blue represent neurons, red line represent the connection between neurons, and the color of each diamond indicates the distances of neurons, from yellow to black. It is generally more darker colored content the distance between neurons are longer.

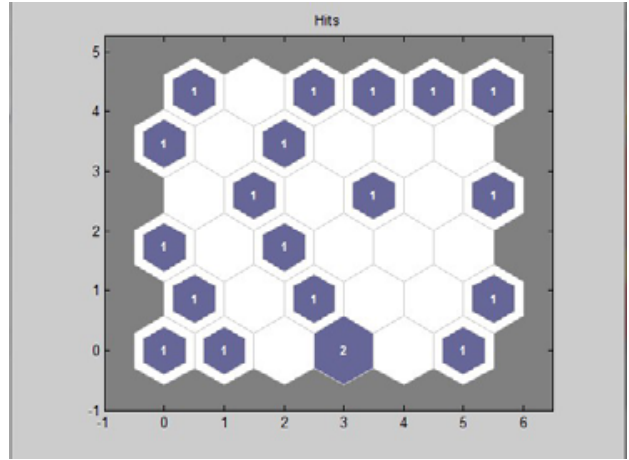

Figure 2. Classification of neurons

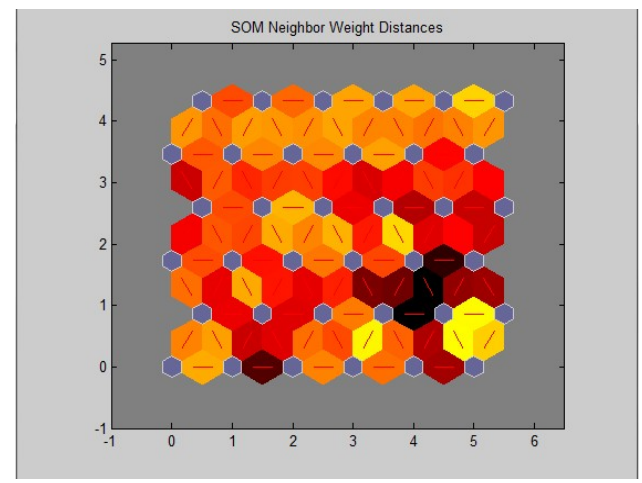

Figure 3. The distance between neurons

Table 3 shows the classification results of the last ten sample data. From the table 4 clustering result data, the first set of data obtained the clustering results for the second class, but the second class is not in completed training classification results. Now, through the space quantization error method based on the deviation from the normal characteristics of the data, we can determine whether this group data is under control or not. Some data may have the same distance with two or more neurons which can not judge the category. Then, by determining the minimum quantization error value, we can solve this problem. When the manufacturing process is out of control, the system is able to make a rapid alarm response, to facilitate timely recovery of stability.

Table 3 Classification result of the test data

\begin{tabular}{ccccccccccc}
\hline Columns & 1 & 2 & 3 & 4 & 5 & 6 & 7 & 8 & 9 & 10 \\
Clustering results & 2 & 8 & 9 & 31 & 2 & 20 & 29 & 13 & 34 & 1 \\
\hline
\end{tabular}

\section{CONCLUSION}

SOM is a popular neural net-work based on unsupervised learning, which means that it is not necessary to provide the network with an expected output during the training period. SOM networks' ability is to associate new data with similar previously learnt data can be applied to forecasting applications. So we can identify the classification of new products by learning the controlling products data in the cement slide shoe bearing the manufacturing process. Once SOM neural network training 
complete through the above self-learning classification arithmetic by collecting enough samples to train, the type of defects of sample which is input to network can be able to diagnose. So it is able to make a rapid alarm response, to facilitate timely recovery of stability

\section{ACKNOWLEDGMENT}

This paper was supported by the Project of National Natural Science Foundation of China (No.71171154), the Fundamental Research Funds for the Central Universities (No. 133204005).

\section{REFERENCES}

[1] Jianbo Yu, Lifeng Xi. Using MQE Chart Based on Self-Organizing Map Neural Network for Monitoring Out-of-control Signals in Manufacturing Processes[J]. International Journal of Production Research, 2008, vol46, no21: 5907-5933

[2] Niaki S T A, Davoodi M. Designing a multivariaterm ultrstage quality control system using artificial nueral networks[J]. International Journal of Production Research, 2009, 47(1): 251-271

[3] M. Lópeza, S. Valero, C. Senabre, J. Aparicio, A. Gabaldon. Application of SOM neural networks to short-term load forecasting:
The Spanish electricity market case study[J]. Electric Power Systems Research, 2012, no91: 18-27

[4] REN Jun-hao, JI Pei-qi, GENG Yu. Application and improvement of SOM network in remote sensing image classification[J]. Application Research of Computers, 2011, vol28, no3: 1170-1173

[5] Belzarena, Pablo; Aspirot, Laura. End-to-end quality of service seen by applications: A statistical learning approach[J]. Computer Networks, 2010, vol54, n17: 3123-3143

[6] Correa, RF; Ludermir, TB. Semantic Mapping and K-means applied to Hybrid SOM-Based Document Organization System Construction[C]. Proceeding of the 23rd annualacm symposium on applied computing, 2008(5): 1112-1116

[7] El-Midany, T.T.; El-Baz, M.A.; Abd-Elwahed, M.S. A proposed framework for control chart pattern recognition in multivariate process using artificial neural networks[J]. Expert Systems with Applications, 2010, vol37, no2, 1035-1042

[8] Wang Hai-yu. Statistical quality monitoring of multiple steps delay feedback controlled process[J]. Systems Engineering - Theory \& Practice, 2011, vol31, no1: 138-143

[9] XIA Ling, GU Han-yu. SOM-based approach for addressing multicriteria scheduling problems[J]. Computer Integrated Manufacturing Systems, Vol4, No4, 2008(4): 757-761 